\title{
Prevalence of Drug Prescriptions and Potential Safety in Patients with Cirrhosis: A Retrospective Real-World Study
}

\author{
Rianne A. Weersink ${ }^{1,2} \cdot$ Katja Taxis $^{2}$. Joost P. H. Drenth ${ }^{3} \cdot$ Eline Houben $^{4} \cdot$ Herold J. Metselaar $^{5}$. \\ Sander D. Borgsteede ${ }^{1,6}$
}

Published online: 24 October 2018

(c) The Author(s) 2018

\begin{abstract}
Introduction Patients with cirrhosis are at risk for adverse drug reactions (ADRs) due to altered pharmacokinetics and pharmacodynamics. We aimed to determine the prevalence of drug prescriptions and the potential safety of these prescriptions in a real-world cohort of patients with cirrhosis.

Methods This was a retrospective cohort study based on linked real-world data from the Out-patient Pharmacy Database and the Hospitalisation Database of the PHARMO Database Network. Patients with a diagnosis of cirrhosis between January 1998 and December 2015 were included. Follow-up ended when the patient underwent a liver transplant, died, transferred out of the database, or on 31 December 2015. Prescription data were derived from a community pharmacy database and were compared with our previously developed safety recommendations for 209 drugs.

Results In total, 5618 patients were included and followed for a median of 3 years (interquartile range [IQR] 1-7). In the first year after the diagnosis, patients used a median of nine drugs (IQR 5-14), with proton pump inhibitors (prevalence 53.9\%), aldosterone antagonists (43.6\%), and sulfonamide diuretics (41.3\%) being the most commonly used drug groups. Almost half (48.3\%) of 102,927 prescriptions consisted of drugs with a safety recommendation. The prevalence of potentially unsafe drug use was $60.0 \%$ during the total follow-up. Three nonsteroidal anti-inflammatory drugs (NSAIDs) were among the five most commonly used potentially unsafe drugs.

Conclusions Patients with cirrhosis use a large number of drugs. Almost two-thirds of patients in our cohort used potentially unsafe drugs. To prevent ADRs in these frail patients, personalised pharmacotherapy is necessary.
\end{abstract}

Electronic supplementary material The online version of this article (https://doi.org/10.1007/s40264-018-0744-1) contains supplementary material, which is available to authorized users.

Rianne A. Weersink

r.a.weersink@rug.nl

1 Department of Clinical Decision Support, Health Base Foundation, Papiermolen 36, 3994 DK Houten, The Netherlands

2 Department of Pharmacy, Unit of Pharmacotherapy, -Epidemiology and Economics, University of Groningen, Antonius Deusinglaan 1, 9713 AV Groningen, The Netherlands

3 Department of Gastroenterology, Radboud University Medical Center, PO Box 9101, 6500 HB Nijmegen, The Netherlands
4 PHARMO Institute for Drug Outcomes Research, Van Deventerlaan 30-40, 3528 AE Utrecht, The Netherlands

5 Department of Gastroenterology and Hepatology, Erasmus University Medical Center, PO Box 2040, 3000 CA Rotterdam, The Netherlands

6 Department of Hospital Pharmacy, Erasmus University Medical Center, PO Box 2040, 3000 CA Rotterdam, The Netherlands 


\section{Key Points}

Patients with cirrhosis have an increased risk of adverse drug reactions due to pharmacokinetic and pharmacodynamic changes, and the large number of medications they use.

Potentially unsafe drug use is common in patients with cirrhosis and more efforts are needed to decrease the use of these drugs in these patients.

Clinical decision support can be used to alert healthcare professionals when prescribing potentially unsafe drugs in patients with cirrhosis.

\section{Introduction}

Worldwide, approximately 1 million people die every year of liver cirrhosis [1]. Cirrhosis develops due to persistent liver damage caused by chronic liver diseases. Patients usually require several medications for the treatment of their disease and its complications, but few studies assessed drug utilization in cirrhotic patients [2-4]. Almost $30 \%$ of patients with cirrhosis suffer from adverse drug reactions (ADRs) [2]. The risk of ADRs is exacerbated as a result of hepatic impairment influencing drug pharmacokinetics and pharmacodynamics [5, 6]. For example, exposure to pantoprazole is fiveto eightfold increased in patients with cirrhosis compared with healthy controls due to decreased hepatic clearance $[7,8]$. Likewise, the use of nonsteroidal anti-inflammatory drugs (NSAIDs) increases the risk for renal impairment [9, 10]. Patients with cirrhosis therefore require personalised pharmacotherapy.

Recently, we published practical guidance for over 200 drugs to support healthcare professionals in prescribing drugs to patients with cirrhosis [11]. To prioritize the remaining drugs to be evaluated and to explore potential improvements for safe prescribing in these patients, current drug use needs to be mapped. An evaluation of prescribing in cirrhosis was the subject of two previous studies [12,13], both of which were hospital-based, cross-sectional studies that compared prescriptions with data from the product label and a combination of literature. There is limited knowledge on real-world drug use in primary care, where the risks may be higher due to a wider range of drugs prescribed with lessintensive monitoring. Therefore, the aims of our study were to (1) determine which drugs are most frequently used in a real-world cohort of patients with cirrhosis, and (2) compare real-world drug use with our previously elaborated practical guidance on safe drug use in cirrhosis [11].

\section{Methods}

\subsection{Study Type and Data Source}

This was a retrospective cohort study based on linked data from the Out-patient Pharmacy Database and the Hospitalisation Database of the PHARMO Database Network [14]. This population-based network of healthcare databases combines data from different primary and secondary healthcare settings. The Out-patient Pharmacy Database contains data on general practitioner- and specialist-prescribed healthcare products, dispensed by community pharmacies, while the Hospitalisation Database comprises information on hospital admissions, including diagnoses of hospital discharges, from the national Dutch Hospital Data Foundation [15]. Together, these databases cover an area of almost 4 million residents of The Netherlands, representative of the total population of The Netherlands.

\subsection{Study Population}

The Hospitalisation Database was used to include patients with a diagnosis of cirrhosis between January 1998 and December 2015. They were selected by International Classification of Diseases, Ninth Revision (ICD-9) codes 571.2 or 571.5, and, after 2013, by ICD Tenth Revision (ICD-10) codes K70.3 or K74.6. The index date was the date of discharge from hospital with a diagnosis of cirrhosis. Patients younger than 18 years of age at the index date, and patients with a liver transplant before the index date, were excluded. Patients who did not receive a single drug dispensing after the index date were also excluded as they were probably lost to follow-up. Follow-up was ended when the patient underwent a liver transplant (ICD-9 code V42.7, ICD-10 code Z94.4), transferred out of the database because of death or another reason, or on 31 December 2015, whichever occurred first. From the included patients, all dispensed drugs during the total follow-up were extracted from the Out-patient Pharmacy Database. Available data from the Hospitalisation Database were the ICD coding of cirrhosis per patient, his or her date of discharge with the diagnosis of cirrhosis, and, if applicable, the date of a liver transplantation.

\subsection{Analyses}

To determine the most frequently used drugs, we calculated the period prevalence of drug use in the first year of follow-up and during the total follow-up. For this, we divided the number of drug users per period by the total number of patients during that period. Drugs were categorized into 
subgroups according to the Anatomical Therapeutic Chemical (ATC) classification system (4th level) [16]. ATC subgroups were counted only once per patient. We listed the 10 most frequently used ATC subgroups and their main individual drugs (ATC 5th level). Of these 10 most frequently used subgroups, the period prevalence per calendar year from 1998 to 2015 was also determined. For every calendar year, the number of users per subgroup was divided by the total number of patients in the database in that year.

The potential safety of drug use was assessed using the practical guidance we recently published on safe prescribing for 209 drugs in patients with cirrhosis [11]. In this guidance, every drug was classified according to potential safety risks (i.e. safe, no additional risks known, additional risks known, unsafe, and unknown), and, if applicable, specific dosing advice was also given. For some medications, the safety class depends on the severity of cirrhosis (Child-Pugh class [17]). Since no data were available in the database regarding the severity of cirrhosis, these drugs could not be classified further. Drugs not included in the practical guidance were classified as 'safety not yet evaluated'. Per safety class, the period prevalence of drug use during the total follow-up was determined. We also listed the five most frequently used drugs per safety class. Because no data on clinical outcomes were available, we only assessed 'potential safety' of drug use.

Descriptive statistics were used to describe the characteristics of the study population. All analyses were performed using Statistical Package for the Social Sciences (SPSS) version 24 (IBM Corporation, Armonk, NY, USA), and the Strengthening the Reporting of Observational Studies in Epidemiology (STROBE) statement was used to report this study.

\section{Results}

\subsection{Patient Characteristics}

In total, 5618 patients with cirrhosis were included (Fig. 1). Characteristics of the study population are shown in Table 1. Males represented $59.4 \%$ of patients and the mean age was 60.7 years (SD 12.5). Almost half of the patients (49.5\%) suffered from alcoholic cirrhosis, while the cause of cirrhosis was different or unspecified in $50.5 \%$ of patients.

\subsection{Characteristics of Prescriptions}

In total, there were 102,927 prescriptions written during the total follow-up. In the first year of follow-up, patients used a median of nine different drugs (interquartile range [IQR] 5-14). Analysing the duration of use, $37.6 \%$ of drugs were dispensed only once, $15.2 \%$ were administered twice,

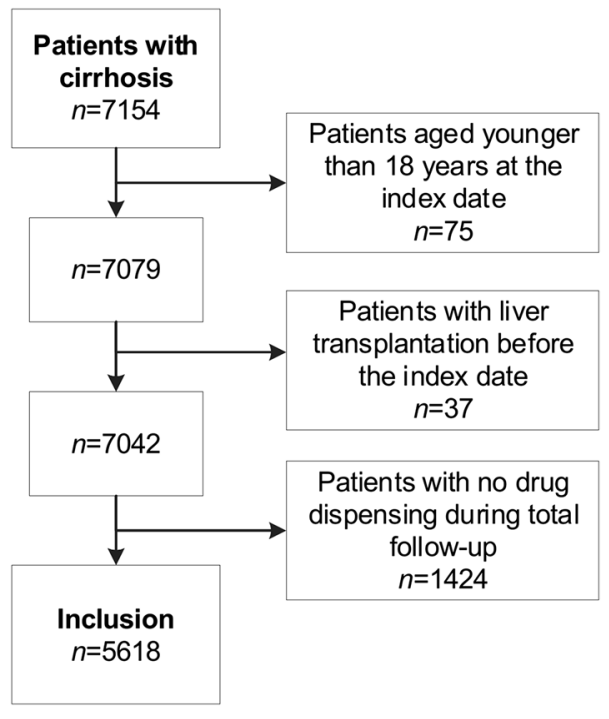

Fig. 1 Study inclusion process

and the remaining $(47.2 \%)$ were administered more often (median 2; IQR 1-7). General practitioners wrote $61.5 \%$ of prescriptions, specialists wrote $36.4 \%$ of prescriptions, and for $2.2 \%$ of prescriptions, the prescriber was either another healthcare professional or was unknown.

Table 2 shows the most frequently used drug groups during the total follow-up, with their main individual drugs. Proton pump inhibitors and diuretics were most often used. The period prevalence of use of these drug groups was also determined per calendar year from 1998 to 2015 (see electronic supplementary material Fig. 1). Period prevalence of most groups slightly decreased over time, while usage of proton pump inhibitors steadily increased from approximately 30\% in 1998 to almost $40 \%$ in 2015 .

\subsection{Assessment of Potential Safety}

Of the 102,927 prescriptions, $48.3 \%$ consisted of one of the 209 drugs that carried a safety recommendation according to [11], while the safety of the remaining prescriptions (51.7\%) has not yet been evaluated. During the total followup, almost all patients used at least one drug for which safety has not yet been evaluated (96.4\%) (Table 3). Furthermore, almost $40 \%$ (39.9\%) of patients used a drug for which the limited clinical data do not allow safety to be determined ('unknown'). More than two-thirds (68.9\%) of patients used a drug for which safety depends on the severity of cirrhosis (Child-Pugh class [17]).

Potentially unsafe drugs were used by $60.0 \%$ of patients during the entire follow-up. Most patients used one of these drug (35.7\% of patients), but $24.3 \%$ used multiple drugs (range 2-8). Examining the duration of use, $63.3 \%$ of potentially unsafe drugs were dispensed more than once (median 
Table 1 Characteristics of the study population

\begin{tabular}{|c|c|c|c|}
\hline & Total & Alcoholic cirrhosis & $\begin{array}{l}\text { Other or } \\
\text { unspecified } \\
\text { cirrhosis }\end{array}$ \\
\hline No. of patients & $5618(100.0)$ & $2782(100.0)$ & $2836(100.0)$ \\
\hline \multicolumn{4}{|l|}{ Sex } \\
\hline Male & $3339(59.4)$ & $1810(65.1)$ & $1529(53.9)$ \\
\hline Female & $2279(40.6)$ & $972(34.9)$ & $1307(46.1)$ \\
\hline Age, years $[$ mean $\pm S D]$ & $60.7 \pm 12.5$ & $58.4 \pm 10.5$ & $62.9 \pm 13.9$ \\
\hline Years of follow-up [median (IQR)] & $3(1-7)$ & $4(1-8)$ & $3(1-7)$ \\
\hline \multicolumn{4}{|l|}{ Reason for end of follow-up } \\
\hline Liver transplantation & $179(3.2)$ & $56(2.0)$ & $123(4.3)$ \\
\hline 31 December 2015 & $3278(58.3)$ & $1650(59.3)$ & $1628(57.4)$ \\
\hline Other (died or transferred out of the database) & $2161(38.5)$ & $1076(38.7)$ & $1085(38.3)$ \\
\hline Unique drug prescriptions in the first year of follow-up [median (IQR)] & $9(5-14)$ & $8(5-12)$ & $9(5-14)$ \\
\hline Unique drug prescriptions during total follow-up [median (IQR)] & $28(18-43)$ & $26(16-39)$ & $31(20-46)$ \\
\hline
\end{tabular}

Data are expressed as $n(\%)$ unless otherwise specified

Unique drug prescriptions were the number of different individual drugs prescribed (ATC 5th level)

$I Q R$ interquartile range, $S D$ standard deviation, ATC Anatomical Therapeutic Chemical

2; IQR 1-8). General practitioners prescribed $62.6 \%$ of potentially unsafe drugs, specialists prescribed $33.3 \%$, and the prescriber was either another healthcare professional or was unknown in $4.2 \%$ of prescriptions. Pantoprazole was the most commonly used potentially unsafe drug, while three NSAIDs were in the top five (Table 3).

\section{Discussion}

This study described drug utilization in a large, real-world cohort of patients with cirrhosis. We demonstrated that patients with cirrhosis used a median of nine drugs in the year after the diagnosis, with proton pump inhibitors and diuretics being the most commonly used drug groups. Nearly half of the prescriptions consisted of drugs with a safety advice, and the potential safety of these prescriptions were further assessed. Almost two-thirds of patients used a potentially unsafe drug during the study period, while $40 \%$ used a drug for which safety was never clinically studied in patients with cirrhosis.

Two hospital-based studies examined drug utilization in cirrhosis on a smaller scale. The first was a Spanish multicentre prospective study in a cohort of a little over 500 admitted patients $[3,4]$, while the second was a retrospective chart study in a Swiss university hospital among 400 patients $[2,13]$. We found a comparable prevalence of drug use for complications of cirrhosis (i.e. diuretics, $\beta$-blockers and osmotically acting laxatives); however, the use of NSAIDs, benzodiazepines and glucocorticoids seemed higher in our cohort. A possible explanation for this is that the largest part of prescriptions in our study came from general practitioners, i.e. primary care. In addition, the proportion of proton pump inhibitor use appeared higher in our study, which is probably due to the increasing prevalence of proton pump inhibitor use found over time [18, 19].

We found a period prevalence of $60 \%$ potentially unsafe drug use during the total follow-up. As we only assessed potential safety for half of the prescriptions, potentially unsafe drug use is possibly more prevalent. NSAIDs were frequently prescribed, however they increase the risk of renal dysfunction and gastrointestinal bleeding in patients with cirrhosis and therefore should be avoided [11, 13]. Healthcare professionals are often not aware of these risks and are afraid of prescribing paracetamol because of possible hepatotoxicity [20,21]. The prevalence of diclofenac use was indeed higher than paracetamol use in our study. While paracetamol does cause hepatotoxicity when overdosing, conversion to the reactive hepatotoxic metabolite is not increased in cirrhosis but possibly even decreased due to reduced enzyme activity [22]. Research is limited in decompensated patients with other risk factors for hepatotoxicity (malnourishment or chronic alcohol abuse) and a dose reduction may be warranted [22]. Pantoprazole and atorvastatin are potentially unsafe drugs also commonly used. For both drugs, large pharmacokinetic alterations occur in patients with cirrhosis (five- to sevenfold increases in exposure $[8,23])$, and in our practical guidance we therefore recommended using another drug from the same group without these large increases in exposure (e.g. esomeprazole or rosuvastatin) in order to prevent ADRs [7, 24, 25]. Almost $40 \%$ of patients used a drug classified as 'unknown', 
Table 2 Period prevalence of the 10 most frequently used drug groups in the first year of follow-up and during total follow-up, with their main individual drugs as example

\begin{tabular}{|c|c|c|c|c|c|}
\hline \multirow[t]{2}{*}{ Drug group } & \multirow[t]{2}{*}{ ATC code } & \multicolumn{2}{|c|}{ First year of follow-up } & \multicolumn{2}{|c|}{ Total follow-up } \\
\hline & & No. of users & $\begin{array}{l}\text { Period preva- } \\
\text { lence }(\%)^{\mathrm{a}}\end{array}$ & No. of users & $\begin{array}{l}\text { Period } \\
\text { prevalence } \\
(\%)^{\mathrm{a}}\end{array}$ \\
\hline Proton pump inhibitors & A02BC & 3030 & 53.9 & 3833 & 68.2 \\
\hline Pantoprazole & $\mathrm{A} 02 \mathrm{BC} 02$ & 1404 & 25.0 & 1963 & 34.9 \\
\hline Omeprazole & A02BC01 & 1149 & 20.5 & 1809 & 32.2 \\
\hline Sulfonamide diuretics, plain & $\mathrm{C} 03 \mathrm{CA}$ & 2322 & 41.3 & 2800 & 49.8 \\
\hline Furosemide & C03CA01 & 2001 & 35.6 & 2470 & 44.0 \\
\hline Osmotically acting laxatives & A06AD & 1832 & 32.6 & 2791 & 49.7 \\
\hline Lactulose & A06AD11 & 1155 & 20.6 & 1661 & 29.6 \\
\hline Macrogol, combinations & A06AD65 & 804 & 14.3 & 1623 & 28.9 \\
\hline Aldosterone antagonists & C03DA & 2451 & 43.6 & 2755 & 49.0 \\
\hline Spironolactone & C03DA01 & 2428 & 43.2 & 2734 & 48.7 \\
\hline Benzodiazepine derivates, anxiolytics & N05BA & 1216 & 21.6 & 1763 & 31.4 \\
\hline Oxazepam & N05BA & 741 & 13.2 & 1121 & 20.0 \\
\hline Benzodiazepine derivates, hypnotics and sedatives & N05CD & 1125 & 20.0 & 1638 & 29.2 \\
\hline Temazepam & N05CD07 & 869 & 15.5 & 1302 & 23.2 \\
\hline Anilides & N02BE & 906 & 16.1 & 1592 & 28.3 \\
\hline Paracetamol & N02BE01 & 637 & 11.3 & 1199 & 21.3 \\
\hline Beta-blocking agents, non-selective & C07AA & 1215 & 21.6 & 1465 & 26.1 \\
\hline Propranolol & C07AA05 & 1146 & 20.4 & 1354 & 24.1 \\
\hline Combinations of penicillins, incl. beta-lactamase inhibitors & J01CR & 538 & 9.6 & 1416 & 25.2 \\
\hline Amoxicillin and enzyme inhibitor & J01CR02 & 538 & 9.6 & 1416 & 25.2 \\
\hline Glucocorticoids & $\mathrm{H} 02 \mathrm{AB}$ & 726 & 12.9 & 1413 & 25.2 \\
\hline Prednisolone & H02AB06 & 511 & 9.1 & 982 & 17.5 \\
\hline
\end{tabular}

Drug groups are defined as ATC 4th-level subgroups, and individual drugs are defined as ATC 5th-level drugs. Drugs are sorted in descending order by the total follow-up prevalence of the drug group

ATC Anatomical Therapeutic Chemical

a Percentages do not round up to $100 \%$ because patients can be counted in more than one group

meaning there was no, or not enough, literature to formulate a recommendation on safety and dosing in cirrhosis. Most prevalent in this class were some important antibiotics (i.e. doxycycline, nitrofurantoin and flucloxacillin). Clinical research is needed to assess the safety of these drugs in cirrhosis. Likewise, almost all patients used a drug for which no safety evaluation was yet available. Based on the findings of this study, prioritization of the subsequent drugs to be evaluated is possible. Benzodiazepines are important candidates based on their high prevalence of use.

Patients in this study were followed for several years to get a complete picture of real-world drug use. We excluded cirrhotic patients without a single drug prescription during the total follow-up as these were most likely patients lost to follow-up, either because they moved away or they received their drugs from a pharmacy outside the database area, and, in some cases, mislinkage may have occurred. Excluding these patients might have resulted in an overestimation of the prevalence of drug use; however, we think it was unlikely that patients with cirrhosis did not receive any medication after hospital discharge and during total follow-up.

Our cohort included a substantial number of cirrhotic patients in The Netherlands. We did not have detailed clinical data, which limited our analyses. A comparison with the prescribed dose was therefore not possible because most dosing recommendations depend on the severity of cirrhosis (Child-Pugh class [17]). Likewise, information on the outcome of treatment (i.e. occurrence of ADRs) would have provided actual safety data. Hence, no assessment of the clinical safety of the drug regimens could be made with these data, and the drug-risk category indicates potential safety risks. Nevertheless, the focus of this study was to provide insight into drug prescriptions and potentially inappropriate drug utilization in these patients on a large scale. A further study using patient charts can examine adherence to the recommendations on a more granular level. In 
Table 3 Overview of drug use per safety class and the five most frequently used drugs per class according to Weersink et al. [11]

\begin{tabular}{|c|c|c|c|c|}
\hline Safety class ${ }^{\mathrm{a}}$ & Drug & ATC code & No. of users & $\begin{array}{l}\text { Period prevalence dur- } \\
\text { ing total follow-up }(\%)\end{array}$ \\
\hline \multirow[t]{6}{*}{ Safe } & Total $(n=27)^{\mathrm{b}}$ & & 4836 & 86.1 \\
\hline & Spironolactone & C03DA01 & 2734 & 48.7 \\
\hline & Furosemide & C03CA01 & 2470 & 44.0 \\
\hline & Lactulose & A06AD11 & 1661 & 29.6 \\
\hline & Amoxicillin and enzyme inhibitor & J01CR02 & 1416 & 25.2 \\
\hline & Propranolol & C07AA05 & 1354 & 24.1 \\
\hline \multirow[t]{6}{*}{ No additional risks known } & Total $(n=50)^{\mathrm{b}}$ & & 4366 & 77.7 \\
\hline & Macrogol, combinations & A06AD65 & 1623 & 28.9 \\
\hline & Tramadol & N02AX02 & 1091 & 19.4 \\
\hline & Esomeprazole & $\mathrm{A} 02 \mathrm{BC} 05$ & 924 & 16.4 \\
\hline & Acetylsalicylic acid & B01AC06 & 789 & 14.0 \\
\hline & Metoclopramide & A03FA01 & 711 & 12.7 \\
\hline \multirow[t]{4}{*}{ Additional risks known } & Total $(n=3)^{\mathrm{b}, \mathrm{c}}$ & & 156 & 2.8 \\
\hline & Azathioprine & L04AX01 & 99 & 1.8 \\
\hline & Methadone & N07BC02 & 52 & 0.9 \\
\hline & Heparin & B01AB01 & 6 & 0.1 \\
\hline \multirow[t]{6}{*}{ Unsafe } & Total $(n=25)^{\mathrm{b}}$ & & 3368 & 60.0 \\
\hline & Pantoprazole & $\mathrm{A} 02 \mathrm{BC} 02$ & 1963 & 34.9 \\
\hline & Diclofenac & M01AB05 & 1246 & 22.2 \\
\hline & Ibuprofen & M01AE01 & 612 & 10.9 \\
\hline & Naproxen & M01AE02 & 592 & 10.5 \\
\hline & Atorvastatin & C10AA05 & 347 & 6.2 \\
\hline \multirow[t]{6}{*}{ Unknown } & Total $(n=27)^{\mathrm{b}}$ & & 2244 & 39.9 \\
\hline & Doxycycline & J01AA02 & 1080 & 19.2 \\
\hline & Nitrofurantoin & J01XE01 & 622 & 11.1 \\
\hline & Flucloxacillin & J01CF05 & 619 & 11.0 \\
\hline & Magnesium hydroxide & A02AA04 & 240 & 4.3 \\
\hline & Pheneticillin & J01CE05 & 160 & 2.8 \\
\hline \multirow{6}{*}{$\begin{array}{l}\text { Depending on the severity of } \\
\text { cirrhosis }^{\mathrm{d}}\end{array}$} & Total $(n=49)^{\mathrm{b}}$ & & 3872 & 68.9 \\
\hline & Omeprazole & A02BC01 & 1809 & 32.2 \\
\hline & Codeine & R05DA04 & 908 & 16.2 \\
\hline & Metoprolol & $\mathrm{C} 07 \mathrm{AB} 02$ & 872 & 15.5 \\
\hline & Simvastatin & C10AA01 & 846 & 15.1 \\
\hline & Fentanyl & $\begin{array}{l}\text { N02AB03 } \\
\text { N01AH01 }\end{array}$ & 594 & 10.6 \\
\hline \multirow[t]{6}{*}{ Safety not yet evaluated } & Total $(n=1005)^{\mathrm{e}}$ & & 5415 & 96.4 \\
\hline & Thiamine (vitamin B1) & A11DA01 & 1374 & 24.5 \\
\hline & Temazepam & N05CD07 & 1302 & 23.2 \\
\hline & Other emollients and protectives & D02AX & 1236 & 22.0 \\
\hline & Oxazepam & N05BA04 & 1121 & 20.0 \\
\hline & Phytomenadione & B02BA01 & 894 & 15.9 \\
\hline
\end{tabular}

The total number of users per safety class is calculated for the total follow-up and includes all drugs from that class, not only the top five. Patients were only counted once in the total number

ATC Anatomical Therapeutic Chemical

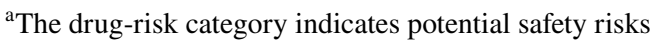

${ }^{b}$ For drugs evaluated by Weersink et al. [11], total numbers represent the number of evaluated drugs prescribed

${ }^{\mathrm{c}}$ This is one of the top three because only three drugs were part of this class

${ }^{\mathrm{d}}$ Since no data were available about the severity of cirrhosis (Child-Pugh class), these drugs could not be classified further

${ }^{\mathrm{e}}$ For drugs with no safety evaluation as yet, the total is the total number of ATC classes without evaluation 
addition, more prospective data on clinical outcomes of drug use in patients with cirrhosis are needed to determine actual safety risk, for example as undertaken by Chalasani et al [26]. Important areas to explore are potential drug-related complications that were attributed to hospital admission or death, such as gastrointestinal bleeding or renal insufficiency attributable to NSAIDs, hepatic encephalopathy or spontaneous bacterial peritonitis linked to proton pump inhibitors, and rhabdomyolysis caused by statins.

How could the prescribing of potentially unsafe drugs in cirrhosis be reduced in practice? Several interventions have shown to be effective in changing prescribing, such as educational outreach visits, audit and feedback and clinical decision support [27, 28]. However, specific applications in cirrhotic patients seem rare. In The Netherlands, the recommendations on safe drug use have been incorporated in clinical decision support systems used by general practitioners and pharmacists and on a free website [29]. To our knowledge, this approach is new for this patient group and can possibly serve as an example to implement personalised pharmacotherapy in clinical practice.

\section{Conclusions}

Our study demonstrated that patients with cirrhosis use many drugs, with proton pump inhibitors and diuretics being the most commonly used drugs. We showed that a large number of cirrhotic patients used potentially unsafe drugs, which possibly puts them at risk for ADRs due to pharmacokinetic or pharmacodynamic changes. Personalised pharmacotherapy is necessary in these patients to prevent ADRs.

Acknowledgements The authors would like to thank all the healthcare providers who contributed information to the PHARMO Database Network.

\section{Compliance with Ethical Standards}

Conflict of interest Eline Houben is an employee of the PHARMO Institute of Drug Outcomes Research, an independent research institute that performs financially supported studies for government and related healthcare authorities as well as several pharmaceutical companies. Rianne Weersink, Katja Taxis, Joost Drenth, Herold Metselaar and Sander Borgsteede declare that they have no competing interests to declare.

Funding This study was financially supported by a grant from the Dutch Digestive Foundation (no. I 10-16). The Dutch Digestive Foundation had no involvement in the study design or collection, and analysis or interpretation of the data.

Ethical approval For this type of study, formal consent is not required.

Availability of data and material Requests for sharing study data must be made on specific grounds either (1) with the aim of corroborating the study results in the interest of Public Health, or (2) in the context of an audit by a competent authority. Sufficient information needs to be provided to confirm that the request is made for one of the abovementioned purposes, including a sound justification and, in case of a request with a view to corroborate study results, a protocol on the research for which the data will be used, or a plan for quality-control checks, as applicable.

Open Access This article is distributed under the terms of the Creative Commons Attribution-NonCommercial 4.0 International License (http://creativecommons.org/licenses/by-nc/4.0/), which permits any noncommercial use, distribution, and reproduction in any medium, provided you give appropriate credit to the original author(s) and the source, provide a link to the Creative Commons license, and indicate if changes were made.

\section{References}

1. Mokdad AA, Lopez AD, Shahraz S, Lozano R, Mokdad AH, Stanaway J, et al. Liver cirrhosis mortality in 187 countries between 1980 and 2010: a systematic analysis. BMC Med. 2014;12:145.

2. Franz CC, Egger S, Born C, Ratz Bravo AE, Krähenbühl S. Potential drug-drug interactions and adverse drug reactions in patients with liver cirrhosis. Eur J Clin Pharmacol. 2012;68:179-88.

3. Lucena MI, Andrade RJ, Tognoni G, Hidalgo R, Sanchez de La Cuesta F. Drug use for non-hepatic associated conditions in patients with liver cirrhosis. Eur J Clin Pharmacol. 2003;59:71-6.

4. Lucena MI, Andrade RJ, Tognoni G, Hidalgo R, Sanchez de La Cuesta F. Multicenter hospital study on prescribing patterns for prophylaxis and treatment of complications of cirrhosis. Eur J Clin Pharmacol. 2002;58:435-40.

5. Verbeeck RK. Pharmacokinetics and dosage adjustment in patients with hepatic dysfunction. Eur J Clin Pharmacol. 2008;64:1147-61.

6. Delco F, Tchambaz L, Schlienger R, Drewe J, Krähenbühl S. Dose adjustment in patients with liver disease. Drug Saf. 2005;28:529-45.

7. Weersink RA, Bouma M, Burger DM, Drenth JPH, Harkes-Idzinga SF, Hunfeld NGM, et al. Safe use of proton pump inhibitors in patients with cirrhosis. Br J Clin Pharmacol. 2018;84:1806-20.

8. Huber R, Hartmann M, Bliesath H, Luhmann R, Steinijans VW, Zech K. Pharmacokinetics of pantoprazole in man. Int J Clin Pharmacol Ther. 1996;34:185-94.

9. Claria J, Kent JD, Lopez-Parra M, Escolar G, Ruiz-Del-Arbol L, Gines P, et al. Effects of celecoxib and naproxen on renal function in nonazotemic patients with cirrhosis and ascites. Hepatology. 2005;41:579-87.

10. Wong F, Massie D, Hsu P, Dudley F. Indomethacin-induced renal dysfunction in patients with well-compensated cirrhosis. Gastroenterology. 1993;104:869-76.

11. Weersink RA, Bouma M, Burger DM, Drenth JPH, Harkes-Idzinga SF, Hunfeld NGM, et al. Evidence-based recommendations to improve the safe use of drugs in patients with liver cirrhosis. Drug Saf. 2018;41:603-13.

12. Ruiz EM, Van De Poll MEC, Brenninkmeijer VJ, Straathof JWA, Derijks LJJ. Drug choice and dosage in patients with severe liver cirrhosis. Pharm Weekbl. 2011;146:135-8.

13. Franz CC, Hildbrand C, Born C, Egger S, Bravo AER, Krähenbühl S. Dose adjustment in patients with liver cirrhosis: impact on adverse drug reactions and hospitalizations. Eur J Clin Pharmacol. 2013;69:1565-73. 
14. Herings RM, Pedersen L. Pharmacy-based medical record linkage systems. In: Strom BL, Kimmel SE, Hennessy S, editors. Pharmacoepidemiology. Hoboken: Wiley; 2012. p. 270-86.

15. Dutch Hospital Data Foundation. Dutch Hospital Data. Available at: https://www.dhd.nl. Accessed 17 Jan 2018.

16. World Health Organization. The anatomical therapeutic chemical classification system with defined daily doses (ATC/DDD). Available at: https://www.whocc.no/atc_ddd_index/. Accessed 15 Jan 2018.

17. Pugh RN, Murray-Lyon IM, Dawson JL, Pietroni MC, Williams R. Transection of the oesophagus for bleeding oesophageal varices. Br J Surg. 1973;60:646-9.

18. Valkhoff VE, Van Soest EM, Sturkenboom M, Kuipers EJ. Timetrends in gastroprotection with nonsteroidal anti-inflammatory drugs (NSAIDs). Aliment Pharmacol Ther. 2010;31:1218-28.

19. Hollingworth S, Duncan EL, Martin JH. Marked increase in proton pump inhibitors use in Australia. Pharmacoepidemiol Drug Saf. 2010;19:1019-24.

20. Rossi S, Assis DN, Awsare M, Brunner M, Skole K, Rai J, et al. Use of over-the-counter analgesics in patients with chronic liver disease. Drug Saf. 2008;31:261-70.

21. Nguyen D, Banerjee N, Abdelaziz D, Lewis J. Trainees' attitudes and preferences toward the use of over the counter analgesics in patients with chronic liver disease. Adv Pharmacoepidemiol Drug Saf. 2014;3:167.
22. Hayward KL, Powell EE, Irvine KM, Martin JH. Can paracetamol (acetaminophen) be administered to patients with liver impairment? Br J Clin Pharmacol. 2016;81(2):210-22.

23. Lea AP, McTavish D. Atorvastatin. Drugs. 1997;53(5):828-47.

24. Weersink RA, Bouma M, Burger DM, Drenth JPH, Hunfeld NGM, Kranenborg M, et al. Evaluating the safety and dosing of drugs in patients with liver cirrhosis by literature review and expert opinion. BMJ Open. 2016;6:e012991.

25. Weersink RA, Drenth JPH, Borgsteede SD. Altered pharmacokinetics of statins explain increased risk of rhabdomyolysis in advanced cirrhosis. Gastroenterology. 2016;151:1036.

26. Chalasani N, Bonkovsky HL, Fontana R, Lee W, Stolz A, Talwalkar J, et al. Features and outcomes of 899 patients with druginduced liver injury: the DILIN prospective study. Gastroenterology. 2015;148(7):1340-52.

27. Ostini R, Hegney D, Jackson C, Williamson M, Mackson JM, Gurman K, et al. Systematic review of interventions to improve prescribing. Ann Pharmacother. 2009;43:502-13.

28. Garg AX, Adhikari NK, McDonald H, Rosas-Arellano MP, Devereaux P, Beyene J, et al. Effects of computerized clinical decision support systems on practitioner performance and patient outcomes: a systematic review. JAMA. 2005;293:1223-38.

29. Expert panel drug safety in liver cirrhosis. Drugs in liver cirrhosis. Available at: https://www.drugsinlivercirrhosis.org. Accessed 15 Jan 2018. 